\title{
LogEasy++: Uma ferramenta para ensino e aprendizagem personalizável utilizando Gamificação
}

\author{
Kelly Suenny M. Correia ${ }^{1}$, Karla Donato Fook ${ }^{2}$, Eveline de Jesus V. Sá ${ }^{1}$ \\ ${ }^{1}$ Departamento de Computação (DComp) \\ Instituto Federal de Educação, Ciência e Tecnologia do Maranhão (IFMA) \\ Campus Monte Castelo - São Luís - MA - Brasil \\ ${ }^{2}$ Divisão de Ciência da Computação (IEC) \\ Instituto Tecnológico de Aeronáutica (ITA) \\ São José dos Campos - SP -Brasil \\ ksuenny@gmail.com, karla@ita.br, eveline@ifma.edu.br
}

\begin{abstract}
This paper presents a customizable teaching and learning tool called LogEasy++, which was developed as a scope expansion of an existing gamified mobile app designed to teach Propositional Logic, called LogEasy. The objective behind the development of LogEasy++ was to produce a support infrastructure for the existing application wich allows its expansion through the customization of the contents to be taught in the app. In order to achieve that, a set of web services and other web develpment technologies were applied.
\end{abstract}

Resumo. Este artigo apresenta uma ferramenta de ensino e aprendizagem customizável chamada LogEasy++, que foi desenvolvida como forma de expandir as funcionalidades de um aplicativo móvel gamificado previamente desenvolvido para ensinar lógica proposicional, o LogEasy. O objetivo do desenvolvimento do LogEasy++ foi produzir uma infraestrutura de suporte para a aplicação existente que permitisse a expansão desta através da customização dos conteúdos a ser ensinados utilizando o aplicativo. Para alcançar este objetivo, foi aplicado um conjunto de tecnologias web, incluindo a tecnologia de serviços web.

\section{Introdução}

O processo de ensino e aprendizagem tem envolvido cada vez mais ferramentas das tecnologias da informação e comunicação. Estas ferramentas são propostas, neste contexto, como uma forma de superar alguns dos desafios inerentes a tal processo. A busca por novas maneiras de despertar o interesse do educando e a viabilização da educação à distância, por exemplo, são alguns destes desafios. Neste sentido, o aprendizado móvel e a gamificação têm sido amplamente utilizados como suporte ao ensino-aprendizagem.

De acordo com [Ozdamli and Cavus 2011], o termo mobile learning (m-learning), ou aprendizado móvel, define um tipo de modelo de aprendizagem que permite que os alunos obtenham materiais de aprendizagem em qualquer lugar e a qualquer momento usando tecnologias móveis e a Internet. O crescimento constante na utilização de dispositivos eletrônicos no processo de aprendizagem pode ser observado nas salas de aula, em ambientes de aprendizagem colaborativos, trabalho de campo, entre outros. $\mathrm{Na}$ área de 
VII Congresso Brasileiro de Informática na Educação (CBIE 2018)

Anais do XXIX Simpósio Brasileiro de Informática na Educação (SBIE 2018)

computação, o aprendizado móvel pode ajudar em uma variedade de disciplinas, já sendo usado, por exemplo, como apoio ao ensino à distância. De acordo com [Yousuf 2007], o m-learning é uma importante ferramenta para o ensino à distância no sentido de fornecer conteúdo, suporte e feedback aos alunos fora da instituição de ensino, além de proporcionar interação entre os estudantes e destes com o tutor.

A gamificação, por sua vez, tem como base a ação de pensar como em um jogo, utilizando as sistemáticas e mecânicas do ato de jogar em um contexto fora de jogo [Busarello et al. 2014]. Segundo [VIANNA et al. 2013], a gamificação abrange a utilização de mecanismos de jogos para a resolução de problemas e para a motivação e o engajamento de um determinado público, o que não significa, necessariamente, a participação em um jogo, mas a utilização dos elementos mais eficientes - como mecânicas, dinâmicas e estética - para reproduzir os mesmos benefícios alcançados com o ato de jogar. A mecânica de um jogo diz respeito à sua funcionalidade, portanto é um elemento fundamental para qualquer ambiente gamificado [Muntean 2011]. De acordo com [ZICHERMANN and CUNNINGHAM 2011], a mecânica de um ambiente de jogo é composta por várias ferramentas capazes de produzir respostas estéticas aos jogadores, entre estas ferramentas encontram-se: Pontos, níveis, placar, divisas, integração, desafios e missões, loops de engajamento, personalização, reforço e feedback.

De acordo com [Muntean 2011], aplicando mecânicas e dinâmicas de jogos a tarefas e processos do e-learning podemos aumentar o engajamento do usuário com uma aplicação e-learning e suas tarefas específicas. Para [Lee and Hammer 2011], a gamificação pode: motivar estudantes a se empenhar na sala de aula, oferecer aos professores ferramentas melhores para guiar e recompensar os estudantes, e fazer com que os estudantes tragam seu potencial completo para a busca do aprendizado.

A aplicação que serviu de base para o desenvolvimento do LogEasy++ foi o LogEasy, uma aplicação móvel local, desenvolvida para o sistema Android, que utiliza gamificação para auxiliar o processo de ensino-aprendizagem de lógica proposicional [Correia et al. 2014]. O projeto se propôs a transformar esta aplicação em uma plataforma de ensino-aprendizagem online e personalizável, chamada LogEasy++. Uma aplicação web, denominada LogEasy++ Professor foi criada. Nela é possível personalizar todo o conteúdo do LogEasy++ de forma que o professor seja capaz de fornecer ao aplicativo todo o conteúdo que deve ser ensinado aos alunos, este conteúdo é disponibilizado no aplicativo em forma de lições, perguntas e respostas, e dicas, que é a forma já utilizada pelo LogEasy para ensinar lógica proposicional.

\section{Trabalhos Relacionados}

A discussão sobre necessidade de mudanças na forma de instrução tradicional e a busca por maneiras de despertar o interesse do educando através da aplicação das novas tecnologias no ambiente educacional têm fomentado o desenvolvimento de diversos trabalhos nesta área. Além disto, a educação à distância possui uma dependência em relação às tecnologias educacionais, que é inerente à sua proposta, o que traz mais um incentivo para o desenvolvimento de tais tecnologias. Neste sentido, muitos projetos têm sido desenvolvidos com o intuito de inserir, cada vez mais, a informática no contexto educacional para melhorar o processo de ensino e aprendizagem, ou mesmo para possibilitar este processo, como é o caso das aplicações de apoio à educação à distância. Considerando este cenário, 
VII Congresso Brasileiro de Informática na Educação (CBIE 2018)

Anais do XXIX Simpósio Brasileiro de Informática na Educação (SBIE 2018)

algumas destas iniciativas serão apresentadas a seguir e terão suas principais características relacionadas aos objetivos deste trabalho.

Kodesh (Koding Shell) é uma plataforma online gamificada com o objetivo de facilitar e estimular a realização de atividades de programação [Campos et al. 2015]. A estratégia de gamificação adotada no Kodesh foi o PBL (Points, Badges and Leaderboards - Pontos, Divisas e Tabelas de Classificação) e seu formato consiste em atividades, que são problemas de programação, compostas de um enunciado e de um conjunto de testes de entrada para o problema a ser desenvolvido pelo aluno e sua correspondente saída esperada. Desta forma, o aluno deve implementar sua solução na própria ferramenta e submetê-la para validação, após a submissão, o aluno recebe um feedback dos erros de compilação, dos testes que falharam ou de sucesso na resolução do problema [Campos et al. 2015].

O Question Mobile consiste em uma ferramenta móvel de avaliação voltada para a EaD (Educação à Distância). Esta ferramenta disponibiliza para o aluno através do aplicativo móvel uma avaliação, na forma de questões de múltipla escolha. Tal avaliação deve ser previamente cadastrada pelo professor a partir de um AVA (Ambiente Virtual de Aprendizagem), que disponibiliza o acesso ao aplicativo Question Mobile, que deverá estar instalado no dispositivo do aluno. Após a resolução da avaliação, o aplicativo envia as respostas do aluno para o AVA, permitindo ao professor avaliar o seu desempenho. Um outro ponto importante desta ferramenta é que, possuindo uma banco de dados local no aplicativo móvel, ele permite o download da avaliação no aparelho para que o aluno possa respondê-la offline e enviar as respostas para o AVA quando houver uma rede disponível para acesso [Fernandes et al. 2012].

Já o Quizz Fis é um produto educacional para tablets e smartphones, desenvolvido para a plataforma Android, com o objetivo de trazer a física para o cotidiano dos alunos. O jogo digital possui três modos: Normal, Survivor e Multiplayer, e foi desenvolvido em formato de quiz (questões de múltipla escolha). O aplicativo utiliza um banco de dados SQLite, no qual foi inserido um banco de questões que abordam os conceitos físicos que têm relação com o cotidiano dos alunos, e permite a edição e a inserção de questões localmente [Silva 2015].

Outra abordagem é o protótipo descrito no artigo Uma Proposta para Apoiar o Ensino e Aprendizagem de Linguagens de Programação publicado em 2017 pela ABED (Associação Brasileira de Educação à Distância). Este trabalho apresenta um protótipo de uma ferramenta de ensino e aprendizagem, móvel, desenvolvida para a plataforma Android, gamificada (utilizando PBL), e disponibilizada na forma de questões de múltipla escolha acerca de uma Linguagem de Programação escolhida. A arquitetura proposta contempla uma ferramenta $w e b$, onde o professor poderá cadastrar os tópicos que serão abordados durante o curso e as questões de cada tópico, além de ter acesso à classificação dos alunos. Os alunos, por sua vez, poderão acessar o aplicativo móvel, que possui login através da internet, e fornece os tópicos previamente cadastrados pelo professor [OLIVEIRA et al. 2017].

\section{LogEasy++}

\subsection{LogEasy}

O LogEasy é o aplicativo móvel que serviu de base para o desenvolvimeno do LogEasy++. O LogEasy foi desenvolvido para a plataforma Android e tem por objetivo utilizar 
VII Congresso Brasileiro de Informática na Educação (CBIE 2018)

Anais do XXIX Simpósio Brasileiro de Informática na Educação (SBIE 2018)

a gamificação para auxiliar o processo de ensino e aprendizagem de lógica proposicional. O conteúdo disponível no aplicativo é composto por: Lições, dicas, perguntas e alternativas, e está organizado em dez níveis, cada nível abordando um tema relacionado ao aprendizado de lógica proposicional. Em termos de gamificação o LogEasy utiliza o sistema PBL (Pontos, Divisas e Quadro de Classificações), sendo que as "divisas"estão relacionadas ao plano de fundo do aplicativo, que utiliza os quatro elementos básicos da natureza (ar, água, fogo e terra) para atribuir ao aluno poderes de dominação relativos a estes elementos cada vez que ele completa um nível.

\subsection{Plataformas}

O LogEasy++ foi desenvolvido para expandir as funcionalidades do aplicativo móvel LogEasy. Esta expansão está relacionada à comunicação do aplicativo móvel com um banco de dados remoto, permitindo a geração de um quadro de classificações contendo vários usuários do aplicativo, não apenas os cadastrados através do mesmo dispositivo. E, além disso, também prevê a criação de uma aplicação web que permite que o cadastro do conteúdo do aplicativo seja feito pelo professor, que é a pessoa mais indicada para elaborar as lições, dicas, questões e alternativas que devem ser utilizadas no aplicativo móvel. Portanto, a arquitetura contempla, além do aplicativo móvel, uma aplicação web para o cadastro dos conteúdos, chamada LogEasy++ Professor, e um serviço de comunicação que permite que as informações inseridas pelo professor sejam carregadas para o dispositivo móvel do aluno. A Figura 1 ilustra a arquitetura do LogEasy++.

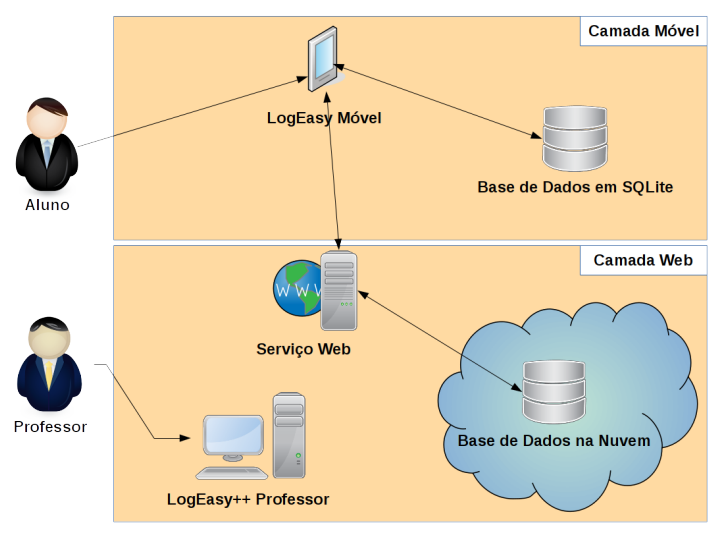

Figura 1. Arquitetura do LogEasy++

Fonte: Elaborado pela própria autora

Arquitetura elaborada para o LogEasy++ conta com: Um banco de dados remoto, que centraliza todas as informações utilizadas nas aplicações que compõem o sistema; Uma aplicação web com funcionalidades relativas ao cadastro e manutenção dos conteúdos que serão disponibilizados, posteriormente, no dispositivo móvel do aluno; Um serviço web que consulta e disponibiliza os dados relativos aos conteúdos cadastrados através da aplicação web, além de sincronizar os dados dos alunos que utilizam o aplicativo e suas pontuações; Um aplicativo móvel, desenvolvido com base no LogEasy, que consome as funcionalidades disponibilizadas pelo serviço web sincronizando o seu banco de dados local com o banco de dados remoto sempre que necessário; e, um banco de dados local no dispositivo móvel que permite o armazenamento dos dados dos alunos e dos 
VII Congresso Brasileiro de Informática na Educação (CBIE 2018)

Anais do XXIX Simpósio Brasileiro de Informática na Educação (SBIE 2018)

conteúdos carregados através do serviço web, permitindo o funcionamento do aplicativo mesmo que não haja conexão com a internet.

\subsection{Aplicação Móvel}

No aplicativo móvel, foi mantido o design do LogEasy, as interfaces foram apenas traduzidas para o português. Além disso, foi feita uma alteração na quantidade de níveis disponíveis no aplicativo. O LogEasy++ possui cinco níveis: Ar, Água, Terra, Fogo e Mestre do Mundo. A seguir, na Figura 2, temos a tela inicial do aplicativo (A), a tela de seleção de usuário (B), a tela de cadastro de usuário (C) e a tela de login (D). Vale lembrar que estas funcionalidades continuam utilizando o banco de dados local do aplicativo, desta forma, continua sendo permitida a utilização do aplicativo mesmo que não haja conexão com a internet.

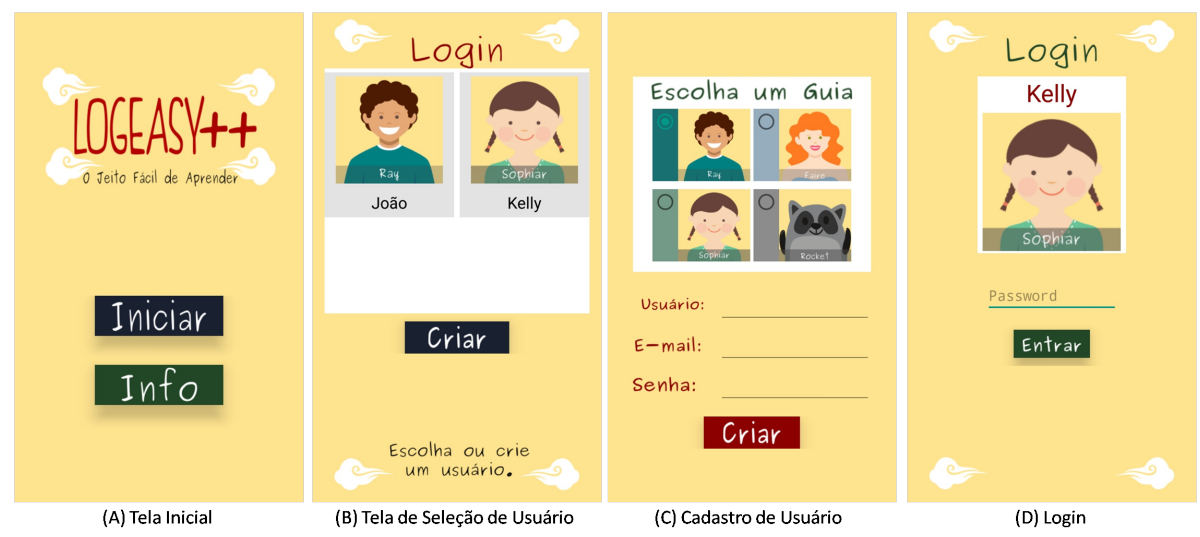

Figura 2. Tela Inicial, Seleção de Usuário, Cadastro de Usuário e Login Fonte: Elaborado pela própria autora

Após o login, o aluno pode fazer o download de um curso para utilizar no aplicativo ou, caso já tenha efetuado o download anteriormente, apenas continuar o curso que já está inserido da base de dados local. Após a seleção do curso o aluno pode fazer a seleção de um nível. A seleção de níveis é liberada conforme a pontuação do usuário. Todos os níveis que exigem uma pontuação menor que a pontuação atual do usuário ficam liberados, assim, o aluno pode sempre rever o conteúdo de um nível anterior. Os níveis que ainda não estão liberados aparecem com uma sombra escura e não é possível selecioná-los. A Figura 3 apresenta a tela de seleção de curso (A) e de seleção de nível (B). 
VII Congresso Brasileiro de Informática na Educação (CBIE 2018)

Anais do XXIX Simpósio Brasileiro de Informática na Educação (SBIE 2018)

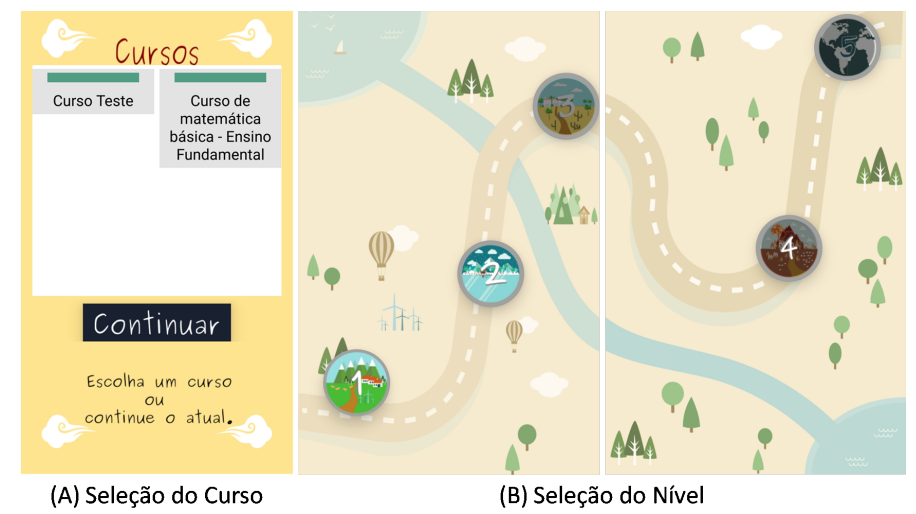

Figura 3. Telas de Seleção de Curso e Nível

Fonte: Elaborado pela própria autora

Após a seleção do nível, o usuário tem à sua disposição a Lição e a Dica relativas ao nível, além da tela de questões. Conforme o usuário muda de nível, o background destas telas também muda, sempre buscando ambientar o elemento correspondente ao nível. A figura 4 mostra as telas referentes ao primeiro nível do aplicativo.

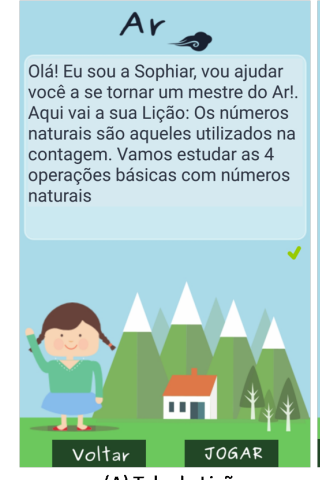

(A) Tela da Lição

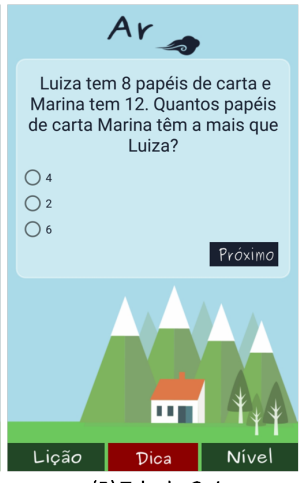

(B) Tela do Quiz

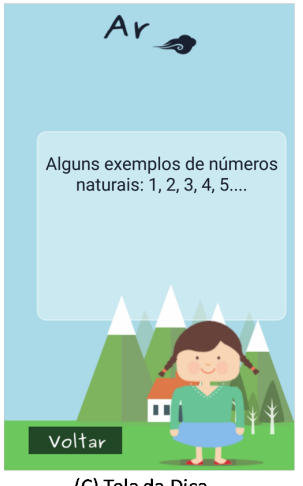

(C) Tela da Dica

Figura 4. Telas de Lição, Questão e Dica do Nível 01

Fonte: Elaborado pela própria autora

O aplicativo possui um menu lateral, que pode ser acessado através de um ícone posicionado no canto superior esquerdo da tela. Através deste menu, o usuário tem acesso às funcionalidades disponíveis no aplicativo. A Figura 5 (A) mostra o menu lateral e, ao lado, temos a tela do perfil do usuário (B), onde é possível visualizar, entre outras informações, os elementos da natureza "dominados"pelo usuário, ou seja, os níveis vencidos.

O aplicativo dispõe ainda de um quadro de classificações, que mostra os alunos que estão realizando o mesmo curso em ordem decrescente de pontuação e crescente de percentual de erros, o percentual de erros funciona como um critério de desempate. Cada vez que o aluno acessa o quadro de pontuações, este é atualizado com os dados dos usuários que estão no banco de dados remoto e, as informações do usuário local são enviadas para o banco remoto, através do serviço web desenvolvido neste trabalho. A Figura 5 (C) mostra o quadro de pontuações do aplicativo. 
VII Congresso Brasileiro de Informática na Educação (CBIE 2018)

Anais do XXIX Simpósio Brasileiro de Informática na Educação (SBIE 2018)

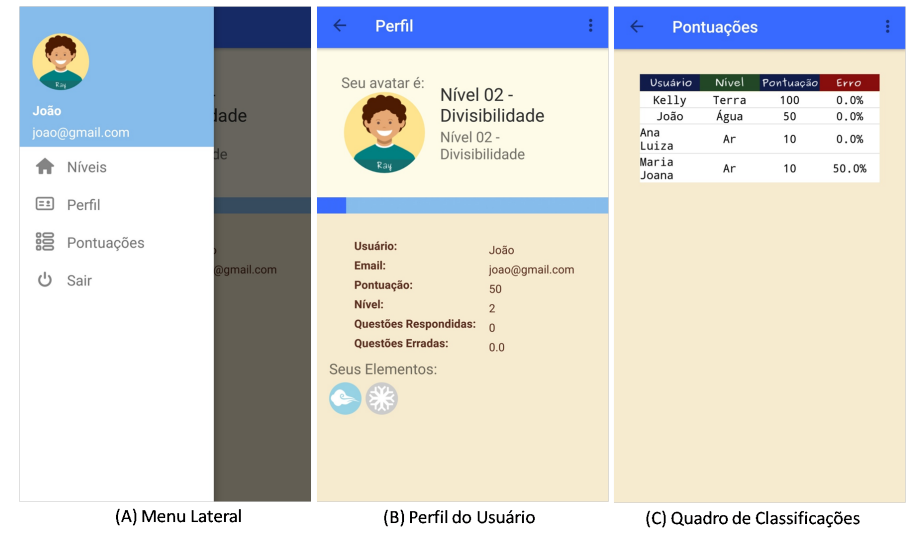

Figura 5. Menu Lateral, Tela de Exibição do Perfil e Quadro de Classificações Fonte: Elaborado pela própria autora

\subsection{Aplicação Web}

A aplicação web desenvolvida, o LogEasy++ Professor, tem o objetivo de permitir o cadastro de todo o conteúdo a ser utilizado do aplicativo. Portanto, temos na aplicação web as funcionalidades de cadastro de: Disciplina, Curso, Conteúdo, Questões e Alternativas, além da visualização, edição e exclusão de todos os itens cadastrados. O cadastro de conteúdos é iniciado pela inclusão de uma disciplina, que é o conceito mais geral, no sentido de área do conhecimento, por exemplo, Língua Portuguesa, Matemática, Lógica etc. A Figura 6 mostra a tela inicial da aplicação (A) e a tela de consulta de disciplinas (B), onde estão disponíveis as opções de visualização, edição e exclusão da disciplina.
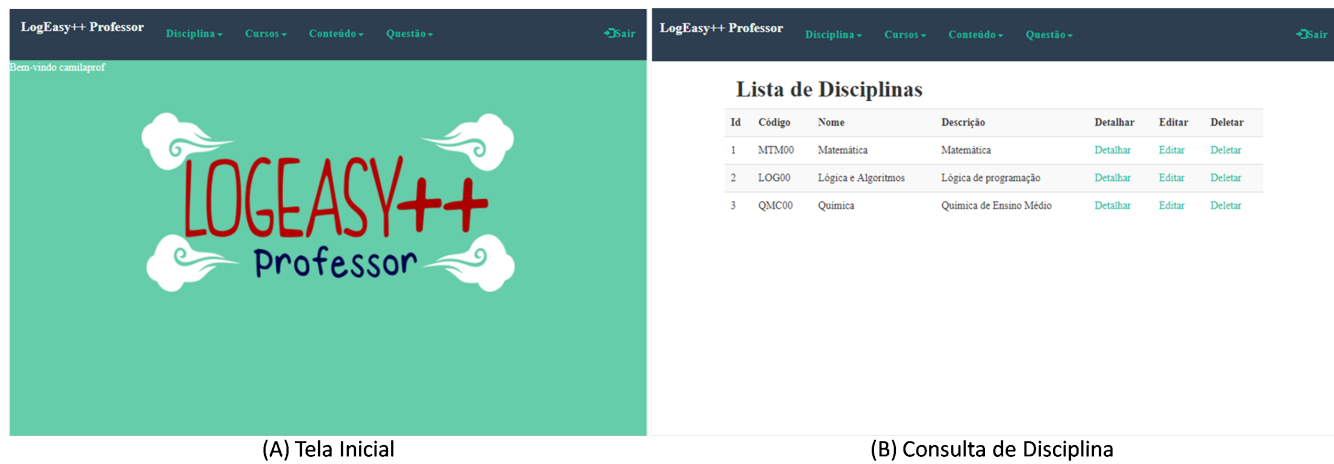

Figura 6. Tela Inicial e Consulta de Disciplinas do LogEasy++ Professor

Fonte: Elaborado pela própria autora

O conceito de curso no LogEasy++ se refere a um conjunto de conteúdos relacionados, cada conteúdo contém uma lição, uma dica e um conjunto de questões. Um curso pode ter até 5 conteúdos diferentes, cada um deles pode abordar um tópico relacionado ao tema do curso ou apresentar níveis de dificuldade diferentes do mesmo tópico. Já o conteúdo representa um nível de aprendizado de um determinado curso. Para criar um conteúdo é necessário selecionar um curso e um nível, que são únicos, para cada curso é permitida a inclusão de apenas um conteúdo em cada nível.

Após a inclusão do conteúdo, é possível cadastrar as questões pertencentes a ele, juntamente com as suas alternativas. Para cada questão é definido um enunciado, a quan- 
VII Congresso Brasileiro de Informática na Educação (CBIE 2018)

Anais do XXIX Simpósio Brasileiro de Informática na Educação (SBIE 2018)

tidade de pontos que ela vale, e até três alternativas, sendo apenas uma verdadeira. Desta forma, a aplicação web LogEasy++ Professor permite o cadastro de cada um dos itens que compõem o conteúdo que será utilizado no aplicativo móvel do aluno, bem como a sua edição. Assim, o professor tem autonomia para elaborar as lições, questões e dicas, além de realizar a organização dos conteúdos em cursos, da forma que ele julgar mais adequada para os seus alunos.

\section{Resultados e Discussões}

Este trabalho descreve uma ferramenta de ensino e aprendizagem gamificada e customizável, o LogEasy++, composta por: um aplicativo móvel gamificado que apresenta os conteúdos a ser estudados em forma de lições, dicas, perguntas e respostas, uma aplicação web para registro dos conteúdos pelo professor, além de um serviço web utilizado para sincronizar os dados utilizados por ambas aplicações.

Foram desenvolvidos a aplicação e o serviço web, já o aplicativo móvel é uma adaptação do LogEasy, uma ferramenta móvel e gamificada aplicada ao ensino e aprendizagem de lógica proposicional. Além disso, foram pesquisados alguns trabalhos relacionados, conforme apresentado na seção 2 com o objetivo de analisar as principais características do LogEasy++ em comparação a aplicativos testados e/ou utilizados em cenários reais. Esta comparação é apresentada a seguir.

As quatro ferramentas apresentadas na seção 2 possuem características em comum com o LogEasy++, entre tais características, cinco foram escolhidas como as que melhor representam o LogEasy++ para a realização de uma análise comparativa entre a aplicação desenvolvida e os trabalhos relacionados apresentados. A Tabela 4 apresenta o resultado da comparação entre as aplicações.

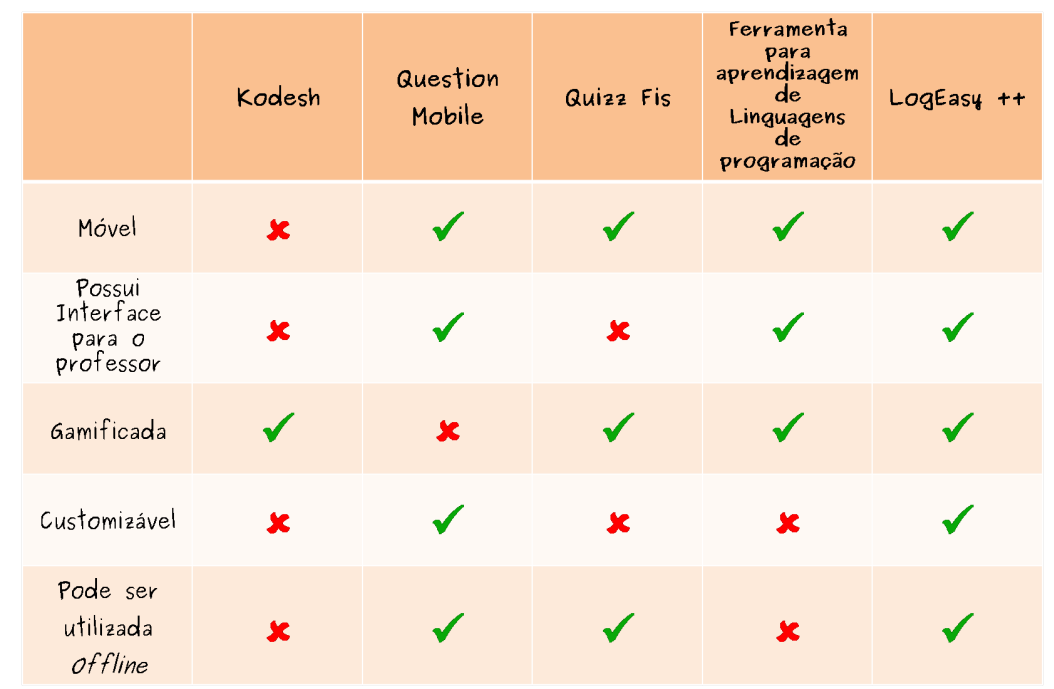

Tabela 1. Tabela comparativa entre o LogEasy++ e os trabalhos relacionados apresentados

Fonte: Elaborado pela própria autora

Os atributos do Logeasy++ destacados na comparação foram os que seguem:

- se a ferramenta é móvel, ou seja, se foi desenvolvido um aplicativo para smartphones e/ou tablets; 
VII Congresso Brasileiro de Informática na Educação (CBIE 2018)

Anais do XXIX Simpósio Brasileiro de Informática na Educação (SBIE 2018)

- se a ferramenta possui uma interface para o professor, onde é possível fornecer conteúdo e/ou acompanhar a evolução do aluno;

- se é gamificada;

- se é customizável, esta característica se refere à possibilidade de utilização da mesma ferramenta para auxiliar a aprendizagem de diversos conteúdos, podendo ser utilizado em diferentes áreas do conhecimento;

- se pode ser utilizado offline, ou seja, se a aplicação utilizada pelo aluno pode funcionar sem conexão com a internet, permitindo que um download seja feito e depois os conteúdos possam ser utilizados localmente.

De acordo com o resultado desta análise comparativa, podemos notar que os atributos comuns às ferramentas pesquisadas e ao LogEasy++ indicam o potencial da ferramenta desenvolvida para ser utilizada no apoio ao processo de ensino e aprendizagem.

Com relação aos trabalhos futuros, destaca-se o estudo de caso que deve ser desenvolvido para validação do LogEasy++ tanto por professores quanto por alunos. Além disso, existem ainda alguns recursos importantes que podem ser implementados. O primeiro que deve ser desenvolvido é um sistema de dupla linguagem para os aplicativos móvel e web, para que professores e alunos possam usar o LogEasy++ em inglês ou português. E, entre outras funcionalidades importantes, pode ser mencionada a implementação de uma interface no LogEasy++ Professor para monitoramento da utilização do aplicativo móvel, onde o professor tenha acesso à pontuação, nível, quantidade de erros, etc. de cada um de seus alunos.

\section{Considerações Finais}

Um dos objetivos deste trabalho foi compreender os principais desafios enfrentados no processo de ensino e aprendizagem, especialmente no que diz respeito ao despertar do interesse dos alunos e à manutenção de seu envolvimento nas atividades escolares. Assim, com base no levantamento bibliográfico realizado, acredita-se que a introdução das tecnologias de informação e comunicação no ambiente educacional, bem como o uso da gamificação, sejam capazes de subsidiar o processo de ensino e aprendizagem de forma a superar os desafios apresentados.

O desenvolvimento do sistema descrito neste trabalho buscou ampliar as funcionalidades de uma ferramenta desenvolvida para apoiar o processo de ensino da Lógica Proposicional, o LogEasy, a fim de convertê-lo em uma ferramenta que pode ser customizada pelo professor, propondo portanto, ajudar o processo de ensino dos mais diversos conteúdos. Esse novo sistema foi chamado LogEasy++ e consiste em um aplicativo móvel, um aplicativo da Web para a configuração de conteúdo e um serviço da Web para comunicação.

Conforme apresentado na seção 2, vários outros projetos de ferramentas de ensino e aprendizagem semelhantes ao LogEasy++ têm sido desenvolvidas, e a comparação, apresentada na seção 4, entre as ferramentas pesquisadas e a descrita neste artigo demonstra a relevância do tema escolhido e a possibilidade de utilização do LogEasy++ para auxiliar o processo de ensino e aprendizagem em cenários reais. 
VII Congresso Brasileiro de Informática na Educação (CBIE 2018)

Anais do XXIX Simpósio Brasileiro de Informática na Educação (SBIE 2018)

\section{Referências}

Busarello, R. I., Ulbricht, V. R., and Fadel, L. M. (2014). A gamificação e a sistemática de jogo: conceitos sobre a gamificação como recurso motivacional. Pimenta Cultural, São Paulo-BR.

Campos, A., Gardiman, R., and Madeira, C. (2015). Uma ferramenta gamificada de apoio à disciplina introdutória de programação. 23 WEI-WORKHOP SOBRE EDUCAÇÃO EM COMPUTAÇÃO.

Correia, K., Cozma, O., Portela, E., and Torres, D. (2014). Android application for learning logic - logeasy application. Technical Report.

Fernandes, K. T., da Trindade, G. O., Ferreira, B., Gomes, A. V., Lucena, M. J. N. R., et al. (2012). Question mobile: Ampliando estratégias de avaliação da aprendizagem por meio de dispositivos móveis. In Anais dos Workshops do Congresso Brasileiro de Informática na Educação, volume 1.

Lee, J. J. and Hammer, J. (2011). Gamification in education: What, how, why bother? Academic Exchange Quarterly.

Muntean, C. I. (2011). Raising engagement in e-learning through gamification. The 6th International Conference on Virtual Learning ICVL.

OLIVEIRA, E. G. d., CAZETTA, P. P., RODRIGUES, G. O., RODRIGUES, M. O., SOLDATI, F. d. P., and FILHO, J. P. (2017). Uma proposta para apoiar o ensino e aprendizagem de linguagens de programaÇÃo.

Ozdamli, F. and Cavus, N. (2011). Basic elements and characteristics of mobile learning. Procedia-Social and Behavioral Sciences, 28:937-942.

Silva, F. U. d. (2015). Uso de quiz em smartphones visando o auxílio na aprendizagem de física no ensino médio.

VIANNA, Y., VIANNA, M., MEDINA, B., and TANAKA, S. (2013). Gamification, Inc.: como reinventar empresas a partir de jogos. MJV Press, Rio de Janeiro-BR.

Yousuf, M. I. (2007). Effectiveness of mobile learning in distance education. Turkish online journal of Distance Education, 8(4).

ZICHERMANN, G. and CUNNINGHAM, C. (2011). Gamification by Design: Implementing Game Mechanics in Web and Mobile Apps. O'Reilly Media, Sebastopol-USA. 